\title{
Bacterial Toxins and Their Modes of Action: A Review Article
}

\author{
Mr. Chala Kenenisa Edae* Dr. Endriyas Kelta Wabalo \\ Department of Biomedical Sciences, Institute of Health, Jimma University, Jimma, Ethiopia
}

\begin{abstract}
Bacterial toxins; the soluble antigens, emitted by various pathogenic microorganisms has a standing notoriety of being a toxin discharged over the span of pathogenesis. Toxins can balance the cell capacities by specifically focusing on various flagging pathways inside the host cell so as to tilt the equalization in microorganisms support. With the upgraded comprehension of the poison refinements strategies, quality cloning, protein arrangements, and three-dimensional crystallographic structures today, we can appreciate the interesting conduct of toxins, for example, restricting capacity to the explicit target cell and a few chemicals capacities. Bacterial pathogens have been causing different infections in people for quite a long time and even today these pathogenic microscopic organisms are coinciding and developing with us, regardless of having diverse means for their control in clinical practices (specifically antimicrobials, immunizations, and phage treatment). Toxins can be grouped into two general classes: exotoxin and endotoxin, and can be delivered by both Gram-positive and Gram-negative microbes. Exotoxins delivered by various microorganisms like botulinum, Clostridium, diphtheria poisons displaying protein exercises like ADP-ribosylation, phospholipase, adenylate cyclase, metalloprotease, deamidase, protease, and deoxyribonuclease action. The vast majority of these microscopic organisms apply their impeding impacts in the contaminated host through toxins. The elucidation of the cellular mechanism of action of the bacterial exotoxins remains a complex problem, but they appear to share a common mechanism of action such as binding to specific receptors on the plasma membranes of the sensitive cells, poreformation, internalization or translocation across the membrane barrier and direct secretion.
\end{abstract}

Keywords: Bacterial toxins; toxin; bacteria

DOI: $10.7176 / \mathrm{JMPB} / 55-03$

Publication date:May $31^{\text {st }} 2019$

\section{BACTERIAL TOXINS}

Pathogenic microscopic organisms and higher eukaryotes have hung out, prompting an exact comprehension of each other's mechanism for infection. Through fast development, microscopic organisms have designed progressively advanced methods to hit precisely where it harms, meddling with crucial host capacities. Nonetheless, toxins are not just valuable to the microscopic organisms - they have additionally turned into fundamental resource for researchers, who would now be able to utilize them as tools to investigate cell forms( 1 ).

Pathogenic microbes apply their destructive impacts in the host through a course of destructiveness factors: exotoxins, endotoxin, intrusions proteins and such. Different poisons emitted by various microorganisms subvert the host intracellular flagging pathways as well as have one of a kind partiality for explicit host cells. Nitty gritty data has been gathered throughout the years with respect to the sanitization, synthetic portrayal, chemical activity and design of different bacterial toxins. Toxins capacity to tie to the explicit target cells makes them a great contender for medication conveyance and in disease therapeutics (2).

Various immunotoxins; half breed particles of bacterial toxinss and antibodies, have wide applications in malignancy and are at present under clinical preliminary. Advances in genomics and proteomics have made an abundance of data identified with the nucleotide and protein successions of various toxins and distinctive databases are accessible with expanding data thereof (3).

One result of the transformative race among host and microbial pathogens is the improvement of advanced and explicit harmfulness factors, which give a particularly preferred standpoint to the life form that produces them. Over the previous decade, our comprehension of the instrument of activity of bacterial poisons has expanded massively. Numerous investigations have added to the sub-atomic definition - and, some of the time, to the revelation - of imperative pathways in cell science. A striking precedent is the finding that lockjaw poison is an endopeptidase, explicit for a layer protein that is restricted on synaptic vesicles and secretory granules. This finding was essential in the meaning of the sub-atomic components that underlie managed emission. Likewise, the significance of guanine-nucleotide-official (G) proteins in flag transduction was revealed by concentrates into the activity of cholera and pertussis poisons, which adjust distinctive classes of G proteins. Since the method of action of numerous poisons has been unwound, they can be utilized as exceedingly explicit and effective apparatuses in cell science (1).

Bacterial poisons; the solvent antigens, discharged by various pathogenic microscopic organisms have a standing notoriety of being a toxic substance emitted throughout pathogenesis. Poisons can adjust the cell capacities by specifically focusing on various flagging pathways inside the host cell so as to tilt the parity in microscopic organisms support. With the upgraded comprehension of the poison filtrations techniques, quality 
cloning, protein arrangements, and three-dimensional crystallographic structures today, we can fathom the extraordinary conduct of poisons, for example, restricting capacity to the explicit target cell and a few catalysts capacities. An academic people group is contributing endeavors to misuses the dazzling highlights of bacterial poisons for various remedial purposes in clinical settings (3).

The bacterial poisons are a noteworthy reason for illnesses since they are in charge of the greater part of indications and sores amid contamination (4). They can be grouped into two classes; I) exotoxins, a dissolvable substance emitted by microorganisms in the host tissues, and ii) endotoxins, by and large living inside the cell divider and discharged into host tissues upon cell demise. The exotoxins demonstration at a separation from the site of contamination and can diffuse through the creature. The explanation of the cell instrument of activity of the bacterial exotoxins remains a mind-boggling issue, however they seem to share a typical component of activity, for example, (I) authoritative to explicit receptors on the plasma layers of the touch cells, (ii) poredevelopment, (iii) disguise or translocation over the film obstruction and iv) coordinate discharge (5).

The exotoxins have an extraordinary proclivity for specific tissues and might be isolated into three classes based on the site influenced: I) neurotoxins follow up on sensory system, ii) enterotoxins on intestinal mucosa and iii) cytotoxins on general tissue. The neurotoxins perceive explicit receptors on the unmyelinated zones of the presynaptic film and repress acetylcholine discharge. Some staphylococcal enterotoxins cause the sustenance harming disorder. The cytotoxins follow up on general tissues; for instance, vacuolating cytotoxin is a standout amongst the most critical harmfulness factors created by Helicobacter pylori, a causative operator of serious gastric illnesses, for example, ulcers and diseases $(6,7)$.

Numerous bacterial poisons are proteins, encoded by the bacterial chromosomal qualities, plasmids or phages. Lysogenic phages shape some portion of the chromosome. The poisons are typically freed from the living being by lysis; however, some are shed with external layer proteins in external film vesicles (8). An imperative non-protein poison is a lipopolysaccharide or endotoxin, which is a constituent of the cell mass of gram-negative microscopic organisms. Poisons may harm the eukaryotic cell film by joining with some basic part, or generally modify its capacity. Numerous poisons consolidate with explicit receptors at first glance layer, every now and again glycoproteins or gangliosides, and enter the cell to achieve their intracellular target. A typical component of a section is absorptive endocytosis. Numerous protein poisons have an A-B structure, B being a polypeptide which ties to the receptor and A being a chemical. Numerous poisons are initiated, either when created by the bacterium or when bound to the film receptor, by proteases (scratching). An enzymatic procedure regular to numerous poisons is adenosine diphosphate (ADP) - ribosylation of the adenylate cyclase administrative proteins, prompting an expansion in intracellular cyclic adenosine monophosphate (cAMP). This is the system of activity of cholera poison. Diphtheria poison catalyzes the exchange of ADP-ribose to stretching factor-2, repressing protein amalgamation. Most poisons follow up on the objective cells to which they tie, however, lockjaw poison, and, to a lesser degree, botulinum poison, rise axons and influence more removed structures. Albeit, numerous poison impacts caused by microscopic organisms have been portrayed just a couple of poisons have been distinguished, described, and their method of action is resolved at the sub-atomic dimension (9).

Bacterial pathogens have been causing different infections in people for quite a long time and even today these pathogenic microscopic organisms are coinciding and developing with us, notwithstanding having distinctive means for their regulation in clinical practices (to be specific antimicrobials, immunizations, and phage therapy)(10).

The vast majority of these microorganisms apply their negative impacts in the contaminated host through poisons. Since the revelation of the principal diphtheria poison from the microbes Corynebacterium diphtheriae, in excess of 500 bacterial poisons are known till date and enhanced comprehension of definite structure, an instrument of activity at cell and organ level is accessible in various distributions (3).

\section{MODES OF ACTION OF BACTERIAL TOXINS}

\section{Plasma membrane permeabilization}

An intermittent topic in cell science is the need to plan frameworks that make it conceivable to access the cytoplasm while keeping the cell alive over the span of the examination. Such a semi-unblemished cell framework can be gotten by treating cells with pore-shaping toxins. These toxins are emitted as water-solvent proteins and, when added to target cells, they tie to cell surface parts going about as receptors (1).

Many pore framing toxins should then oligomerize into an AMPHIPATHIC, roundabout ring-like structure that embeds into natural films to shape a pore6. These structures can be shaped from only a couple of monomers (up to seven), producing little pores, or they might be made out of up to 50 units, making pores sufficiently extensive to allow the section of proteins. The layer spreading over district of these oligomeric edifices is believed to be framed by $\beta$-BARRELS, as appeared by the precious stone structure of the Staphylococcus aureus $\alpha$-poison (9).

The S. aureus $\alpha$-poison and Aeromonas sp. aerolysin5, 8 are the best-portrayed individuals from this family. 
These two poisons are appropriate for making hydrophilic pores of settled size (roughly $1.5 \mathrm{~nm}$ in distance across) that permit trade of little particles and also nucleotides. The $\alpha$-poison has the benefit of being generally utilized; the disadvantage, in any case, is that high dosages in the 100-nM run are typically vital. Portions firmly rely upon the cell type, apparently mirroring the conveyance of an obscure $\alpha$-poison receptor. Conversely, aerolysin has not yet been broadly utilized as an apparatus in cell-permeabilization tests, yet it has the benefit of being compelling on any mammalian cell at picomolar fixations. This is on the grounds that aerolysin ties to glycosylphosphatidylinositol (GPI)- moored proteins, a pervasive class of film related proteins, and restricting happens through the GLYCAN core9 that is available in all the lipid grapples of this protein family (1).

Vast pores, which permit the section of proteins, can be framed by individuals from the cholesteroldependent poison family, which incorporates streptolysin O (SLO) 5, 10. Individuals from this huge poison family require cholesterol for pore development, and they produce channels of variable size, up to $35 \mathrm{~nm}$ in breadth, relating to around 50 subunits. Amid polymerization, every subunit contributes $4 \beta$-strands to the pore-framing $\beta$-barrel, creating a transmembrane structure made out of $\sim 200 \beta$-strands. Without a doubt, short presentation to SLO, trailed by hatching of the cells in a poison free cradle within the sight of $1-2-\mathrm{mM}$ calcium, brought about fix of the film sore. We should, be that as it may, remember that pore-shaping poisons actuate a wide range of cell occasions because of the permeabilization procedure. These incorporate enactment of $G$ proteins, creation of cytokines or vacuolation of the endoplasmic reticulum (ER) (1).

A portion of these impacts are likely activated by particle transitions, calcium section or consumption of cytoplasmic segments, though others are as yet unexplained. These occasions must be considered when deciphering the information from investigations with pore-framing poisons including plasma-film permeabilization of living cells (11).

\section{Labeling explicit atoms}

Toxins are commonly dynamic even at low focuses. This high proficiency is because of the way that most poisons act chemically, bringing about an intensification of cell reactions, and furthermore in lights of the fact that fundamental surface particles on target cells are craftily utilized by the poisons as receptors. This high fondness and particularity for their surface receptors make poisons magnificent tests to pursue the particles with which they associate. Two precedents will be featured here: cholera poison and lysenin, which tie to the ganglioside GM1 and sphingomyelin, individually (3).

The receptor-restricting unit of cholera poison (CT-B) is a homopentamer, in which every subunit can collaborate with ganglioside GM1, a pervasive monosialylated glycosphingolipid found in eukaryotic cells. This pentavalent restricting unit has a higher partiality for bunched GM1, which can be found in cholesterolglycosphingolipid-rich microdomains of the plasma layer called LIPID RAFTS. Lipid pontoons are the subject of escalated explore in view of their job in different central cell procedures, for example, film arranging, flagging and cholesterol homeostasis, and markers for this layer compartment are rare. Thusly, CT-B (marked with peroxidase, colloidal gold or fluorophores) has been a greatly valuable instrument as it permits the location of GM1 on spot smudges, as well as on cells by either electron or fluorescence microscopy $(\boldsymbol{8})$.

Lysenin, a recently described poison, could be likewise valuable to think about lipid pontoons, as it ties explicitly to the pontoon segment sphingomyelin. Little is thought about the intracellular conveyance of sphingomyelin - under typical conditions, as well as under neurotic conditions, such as, lipid-stockpiling maladies. With suitable marking, lysenin ought to permit discovery of sphingomyelin on smudges and also on cells and allow the investigation of the dissemination and transport of this lipid. Up until now, lysenin has been effectively used to choose freak cells that are imperfect in sphingolipid biosynthesis (14).

\section{Evacuating lipids or lipid-tied down atoms}

A few bacterial animal groups create chemicals that influence lipids1, and are in this manner to a great degree helpful in cell science. These incorporate dissolvable phosphatidylinositol-explicit phospholipase Cs (PI-PLCs), the model of which is the catalyst refined from Bacillus cereus. PI-PLCs perceive and divide both phosphatidylinositol and its glucosyl subsidiaries, and are in this manner abused to discharge GPI-secured proteins from the cell surface. This treatment has been the strategy for decision to decide if a protein is GPI-tied down (11).

A below average of compounds of extraordinary intrigue are sphingomyelinases, which cut sphingomyelin to produce ceramide, a second delegate engaged with cell development, separation, apoptosis and layer transport. The coupling of ceramide motioning to explicit flag transduction falls is explicit to both the boost and the cell type, and is by all accounts controlled by the subcellular topology of its creation (16).

Strikingly, transfection with Bacillus cereus sphingomyelinase communicated under the control of an inducible advertiser prompted the ID of a flagging pool of sphingomyelin that is 16 particular from the pool open to exogenous sphingomyelinase (16). 


\section{Examining intracellular courses}

With the prominent exemption of pore-framing toxins referenced above, most bacterial toxins have an enzymatic action towards explicit cytoplasmic targets. This infers the toxin must cross the lipid bilayer and infiltrate the cell. Be that as it may, this occasion once in a while happens at the plasma layer. Poisons for the most part experience endocytosis pursued by transport to explicit intracellular organelles previously they translocate into the cytosol. Particular segments or subunits are engaged with the diverse strides at which these poisons demonstration. The B (authoritative) subunit is engaged with receptor official and translocation into the cytoplasm, though the A (functioning) subunit bears the enzymatic action. On this useful premise, poisons with intracellular targets have been characterized as A- B poisons. Investigations of the courses utilized by these particles ended up being greatly intriguing to cell researcher as they permitted the portrayal of certain vehicle steps, and in some cases even uncovered new intracellular pathways (17).

Toxins have commandeered all realized section focuses into a cell. Some enter through CLATHRINCOATED VESICLES (diphtheria poison, Pseudomonas exotoxin A); others through the putative CAVEOLAR pathway (cholera poison, warm labile enterotoxin from Escherichia coli). Strikingly, the plant poison ricin is disguised by all pathways including a putative clathrin and caveolae free course, most likely as a result of its official to a few receptors. The commitment of poisons to the field of layer transport has, be that as it may, gone a long ways past the underlying disguise step. Investigations of Shiga poison were the first to demonstrate that a particle can be transported from the cell surface to the ER. All the more explicitly, Shiga poison ties to the cell surface by interfacing with globotriaosylceramide (Gb3) and is then endocytosed through clathrin subordinate and clathrin-free systems and directed to the ER through the Golgi device. Regardless of whether Shiga poison stays bound to Gb3 is as yet not known. The correct components that work in this retrograde course appear to be new, and have not yet been completely portrayed $(5,18)$.

Beyond what one retrograde transport pathway could exist, nonetheless, between the Golgi and the ER, as Pseudomonas exotoxin An and cholera poison are likewise transported to the ER through the Golgi, however utilizing a particular system. In this way, these bacterial poisons will keep on being 17 helpful instruments to dismember retrograde transport pathways. Moreover, they have been (and will be) useful in concentrates on the still inadequately comprehended correspondence courses between the endocytic and the biosynthetic pathways, for example, early endosomes to Golgi or plasma film to Golgi Whereas cholera poison can be utilized on any cell type inferable from the omnipresent articulation of its receptor, a few poisons have an amazing cell type explicitness. This is the situation with Shiga poison, which will influence just Gb3-positive cells, for example, those of the resistant framework. Individuals from the clostridial neurotoxin family, which is made out of lockjaw and botulinum neurotoxins, tie at the neuromuscular intersection and experience differential transport in mammalian engine neurons $(1,19)$.

Botulinum neurotoxins remain at the synapse, allowing the study of site-specific endocytosis whereas tetanus toxin is retrograde transported to the cell body and then transcytosed to inhibitory interneurons, making it a marker of choice to study retrograde transport in neurons (G. Lalli and G.S.,unpublished data). In addition, botulinum neurotoxins, which are synthesized as large, multisubunit protein complexes, can be used to follow transcytosis in binding-competent epithelial cells such as colon cells, making use of the fact that these toxins can gain entry into an organism through the intestinal tract $(4,19)$.

The enzymatic activity provided by A-B toxins can also be very useful for altering specific signaltransduction pathways within the cell. In particular, pertussis toxin was instrumental in the discovery of inhibitory heterotrimeric $\mathrm{G}$ proteins, and it is extensively used in the definition of the initial steps in signalling cascades in plant and animal cells. This toxin ADP-ribosylates the $\alpha$-subunits of several G proteins including Gi, Go and $G$ gust, uncoupling them from their cognate receptors. In the case of $G$, this modification causes the silencing of the inhibitory input of the signalling cascade, which induces the indirect activation of downstream effectors, such as adenylyl cyclase, phosphodiesterase and ion channels. In contrast to pertussis toxin, cholera toxin and E. coli enterotoxin ADP-ribosylate activatory G proteins (Gs), including Gt and Golf, by blocking their GTPase activity $(14,22)$.

This leads to the constitutive activation of adenylyl cyclase and the rapid elevation of cyclic AMP levels in the cell. The 'lethal factor' produced by Bacillus anthracis (LF) can also affect signalling, as this zincendopeptidase specifically cleaves several members of the mitogen-activated protein kinase kinase (MAPKK) family, with the notable exception of MAPKK5. Lethal factor elicits the hyper-stimulation of macrophage inflammatory responses and can be used to induce cytokine expression and oxidative burst in these cells. Similarly, the oedema factor produced by the same bacterium transiently increases intracellular cAMP by means of its adenylyl cyclase activity, and modulates the inflammatory immune responses (21).

Inhibition of membrane fusions In addition to their use in studying intracellular transport routes, botulinum and tetanus neurotoxins have been crucial in understanding membrane fusion, in particular during neurotransmitter release. Their catalytic subunits contain a zinc endopeptidase activity that is specific for synaptic members of the SNARE (soluble NSF attachment protein receptor, where NSF stands for N- 
ethylmaleimide-sensitive fusion protein) superfamily: VAMP (vesicleassociated membrane protein), syntaxin and SNAP-25. SNAREs are not only involved in the fusion of synaptic vesicles with the presynaptic plasma membrane, but they are also implicated at all stages of vesicular transport, during which they mediate targeting fidelity and the fusion of lipid bilayers. The action of these toxins is restricted to neurons and neuronally differentiated cells, but cell permeabilization by means of pore-forming toxins or transfection with the active domains of tetanus and botulinum neurotoxins allow the inactivation of specific SNAREs within any cell type $(13,24)$.

To bypass the various cell-type specificities of these toxins, which are imposed by the presence of selective and largely unidentified cell-surface receptors, the catalytic moiety can be directly delivered into the cytoplasm by permeabilizing cells with SLO10. Alternatively, the catalytic portion can be fused to the binding and translocation domains of a toxin with ubiquitously distributed receptors. This strategy has been successful for efficient delivery of $\mathrm{C} 3$ toxin coupled with the binding fragment of $\mathrm{C}$. botulinum $\mathrm{C} 2$ binary toxin (13).

By contrast, the enterotoxin of Bacteroides fragilis does not require entry into the cytoplasm to trigger the reorganization of the actin cytoskeleton. This novel toxin is a metalloprotease, which cleaves E-cadherin on the basolateral side of polarized cells. The specific proteolysis of this essential component of the ZONULA ADHERENS causes the disruption of intercellular junctions, with consequent loss of cell polarization and changes in cell barrier permeability $(1,21)$.

\section{CONCLUSION}

Bacterial toxins are now known as pathogenic agents with their positive considerable applications. Since decades back, they are at the center of life scientists' work in studying disease causations, development of drugs, synthesis of vaccines and antibiotics. Pathogenic bacteria have been causing diseases in humans for centuries and, surprisingly, these pathogens are still coexisting and evolving with us. Despite the improved understanding of their pathogenesis and management, still there is an urgent need to address several unanswered questions like severe humoral response to immunotoxins by host and neutralization of immunotoxins by preexisting antibodies in host. Therapeutic potential of various toxins have been realized in last few decades but further research is required to tap the full potential of the bacterial lethal weapon. Different toxin databases have been created, and are freely available to research community; these databases can enhance our knowledge and understanding in a big way in near future. Bacterial toxins are classified to different classes; endotoxin and exotoxins with subsequent subclasses. The mode of actions of bacterial toxins consists of plasma membrane permeabilizaton, tagging chemicals, affecting cytoskeleton, pore formation, delivery of proteins to cells and interfering with cell signaling.

\section{Disclosure of Conflict of Interest}

All authors declare that there is no conflict of interest and the authors agree to grant the first editing / publishing rights to the journal upon acceptance.

\section{Funding}

This project has no funding source.

\section{Acknowledgement}

None

Originality of the work

This paper is our original work and is not considered for publication in any other place.

\section{REFERENCES}

1. Goot, Giampietro Schiavo and F. Gisou van der, 2001. Bacterial toxin toolkit. nature reviews, pp. 530-538.

2. Sengupta, M.; Austin, S. , 2011. Prevalence and significance of plasmid maintenance functions in the virulence plasmids of pathogenic bacteria. Infect. Immun., Issue 79, p. 2502- 2509.

3. Pankaj Gautam, Pranjali Gupta, Promila Sharma and Pranshu Dangwal, 2015. Virtuous aspects of vicious bacterial toxins. Journal of Chemical and Pharmaceutical Research, 7(6), pp. 279-289.

4. Böhnel H. and Gessler F. ,2005. Botulinum toxins - cause of botulism and systemic diseases?. Vet. Res. Commun., Volume 29, pp. 313-345.

5. Blackall,D. P. and Marques, M. B., 2004. Hemolytic uremic syndrome revisited: Shiga toxin, factor H, and fibrin generation. Am. J. Clin. Pathol., Volume 121, pp. 121, S81-S88.

6. Dinges, M. M., Orwin, P. M. and Schlievert, P. M., 2000. Exotoxins of Staphylococcus aureus.. Clin. Microbiol. Rev., Volume 13, pp. 16-34.

7. Los FC, Randis TM, Aroian RV, Ratner AJ., 2013.Role of pore-forming toxins in bacterial infectious diseases. Microbiol Mol Biol Rev, Issue 77, p. 173-207.

8. Raghava, Sudipto Saha and Gajendra P. S., 2007. prediction of bacterial toxins. Bioinformation Systems, pp. 1-6.

9. Popoff, Blandine Geny and Michel R., 2008. Bacterial protein toxins and lipids:. biology of the cell, pp. 
867-879.

10. Samson, J.E.; Magadan, A.H.; Sabrie, M.; Moineau, S., 2013. Revenge of the phages: Defeating bacterial defences.. Nat. Rev. Microbiol. , Issue 11, p. 675-687.

11. Guidi-Rontani, C., M. Weber-Levy, M. Mock, and V. Cabiaux, 2000. Translocation of Bacillus anthracis lethal and oedema factors across endosome. Cell Microbiol, Issue 2, p. 259-264.

12. Zdeňková, Kamila, 2014. Types of bacterial toxins, biogenic amines and food spoilage microflora, s.l.: European food safety authority.

13. Gonzalez MR, Bischofberger M, Pernot L, Van Der Goot FG, Freche B. , 2008. Bacterialpore-forming toxins: the whole story?. Cell Mol Life Sci, Issue 65, p. 493-507.

14. Provoda, C. J. and Lee, K.-D., 2000. Bacterial pore-forming hemolysins and their use in the cytosolic delivery of macromolecules. Adv. Drug Deliv. Rev. 41, pp. 209-221.

15. Saha, S. and Raghava, G. P. S., 2007. Prediction of neurotoxins based on their function and source. In Silico Biology, pp. 7-25.

16. Middlebrook, J. L. and Dorland, R. B., 1984. Bacterial toxins: cellular mechanisms of action. Microbiol Rev, pp. 199-221.

17. Bhasin, M. and Raghava, G. P. S. , 2004. GPCRpred: an SVM-based method for prediction of familiesand subfamilies of G-protein coupled receptors. Nucleic Acids Res, Volume 32, pp. 383-389.

18. Blower, T.R.; Short, F.L.; Rao, F.; Mizuguchi, K.; Pei, X.Y.; Fineran, P.C.; Luisi, B.F.; Salmond, G.P.C., 2012. Identification and classification of bacterial Type III toxin-antitoxin systems encoded in chromosomal and plasmid genomes. Nucleic Acids Res, Issue 40, p. 6158-6173.

19. Brantl, sabine, 2012. Bacterial type I toxin-antitoxin systems. Bioscience, p. 1488-1490.

20. Makarova, K.S.; Wolf, Y.I.; Koonin, E.V. , 2009. Comprehensive comparative-genomic analysis of type 2toxin-antitoxin systems and related mobile stress response systems in prokaryotes. Biol. Direct, Issue 4, p. 19. 26

21. Marks, J. D. , 2004. Medical aspects of biologic toxins.. Anesthesiol. Clin. North America, Volume 22, pp. 509-532.

22. Michl, P. and Gress, T. M. ., 2004. Bacteria and bacterial toxins as therapeutic agents for solid tumors. Curr. Cancer Drug Targets, Volume 4, pp. 689-702.

23. Nathalie Goeders, Ray Chai, Bihe Chen, Andrew Day and George P. C. Salmond, 2016. Structure, Evolution, and Functions of Bacterial Type III Toxin-Antitoxin Systems. toxins, pp. 1-13.

24. Tamura, B. M. and Chang, B. , 2003. Botulinum toxin: application into acupuncture points for migraine. Dermatol. Surg, Volume 29, pp. 749-754. 